\title{
Transport and Deposition of Fine Mode Particles in Porous Filters
}

\author{
António F. Miguel and A. Heitor Reis
}

Department of Physics, University of Évora, Rua Romão Ramalho, 59, 7000-671 Évora, Portugal; and Évora Geophysics Centre, Apartado 94, 7002-554 Évora, Portugal

E-mail: afm@uevora.pt

\begin{abstract}
Transport and deposition of fine mode aerosol particles in porous filters was investigated analytically. The study was carried out by considering the transport of fine particles through the filter as a convective-dispersive phenomenon. Collector-particle and particle-particle interactions within the filter were considered while reentrainment of deposited particles was assumed to be negligible as compared to particle attachment. Based on these assumptions, transient equations that govern the amount of particles deposited within the filter, pressure drop through the filter, and the filter's permeability and performance are presented. The approach developed here confirms the significance of the Peclet number, the Sherwood number, and geometrical parameters on filtration processes. These results may be useful for filter design as well as for filtering operation monitoring.
\end{abstract}




\section{NOMENCLATURE}

A surface area $\left(\mathrm{m}^{2}\right)$

$\mathrm{c}_{u} \quad$ Cunningham slip correction factor

$C$ particle mass concentration in the airstream within the filter $\left(\mathrm{kg} / \mathrm{m}^{3}\right)$

$C_{0} \quad$ upstream particle concentration $\left(\mathrm{kg} / \mathrm{m}^{3}\right)$

$C_{L}$ downstream particle concentration $\left(\mathrm{kg} / \mathrm{m}^{3}\right)$

$D$ particle diffusion coefficient $\left(\mathrm{m}^{2} / \mathrm{s}\right)$

$I_{p c} \quad$ aerosol particle interception parameter

$k_{B} \quad$ Boltzmannn's constant $\left(\mathrm{kg} . \mathrm{m}^{2} / \mathrm{K} . \mathrm{s}^{2}\right)$

$k_{e} \quad$ excluded surface area factor

$K$ permeability of the filter $\left(\mathrm{m}^{2}\right)$

$L \quad$ thickness of the filter (m)

$l_{c} \quad$ characteristic length of filter collector, e.g., bed, fiber (m)

$p$ pressure $(\mathrm{Pa})$

$\mathrm{Pe} \quad$ Peclet number

$r \quad$ radius (m)

Sh Sherwood number

$t$ time (s)

$T$ absolute temperature (K)

$u \quad$ fluid velocity through the filter $(\mathrm{m} / \mathrm{s})$
$V \quad$ volume $\left(\mathrm{m}^{3}\right)$

\section{Greek Symbols}

$\alpha \quad$ solidity of a new (unloaded) filter

$\varepsilon \quad$ fraction of collectors (e.g., beds, fibers) covered with particles

$\phi$ porosity

$\Phi$ deposition of particles in the filter $\left(\mathrm{kg} / \mathrm{m}^{3}\right)$

$\vartheta$ specific surface area of the filter $\left(\mathrm{m}^{2} / \mathrm{m}^{3}\right)$

$\Theta$ particulate matter transfer coefficient $(\mathrm{m} / \mathrm{s})$

$\Lambda \quad$ filtration coefficient $(1 / \mathrm{m})$

$\mu \quad$ dynamic viscosity of the fluid ( $\mathrm{Pa} \mathrm{s}$ )

$\rho$ density $\left(\mathrm{kg} / \mathrm{m}^{3}\right)$

$\zeta \quad$ ratio of filter volume to particle volume

\section{INTRODUCTION}

Aerosol particles may originate naturally (e.g., dust, salt, pollen, microbes, viruses, etc.) or as a result of industrial activity, incineration, and combustion processes. These particles fall into two categories-fine and coarse mode (Bejan et al., 2004). Fine particles are particles of size less than or equal to $2 \mu \mathrm{m}$ in diameter, while coarse particles are greater than $2 \mu \mathrm{m}$ in diameter.

Fine particles are potentially hazardous for people, electronic equipment, and fine arts (Bejan et al., 2004; Reis et al., 2004; Vafai and Giuliani, 1999). Exposure to the submicrometer fraction of particulate matter has been associated with pulmonary function changes, aggravation of existing respiratory and cardiovascular diseases, and increased susceptibility to respiratory infections, as well as with carcinogenesis and associated mortality (Schwartz and Dockery, 1992; Fleming et al., 1998). In the U.S., air quality standards for particulate matter establish a limit to fine particles in the ambient air of $15.0 \mu \mathrm{g} / \mathrm{m}^{3}$ annual arithmetic mean concentration, and $65 \mu \mathrm{g} / \mathrm{m}^{3}$ daily average concentration (U.S. EPA, 1997).

The majority of the technologies available for removing particles from airstreams fail to capture fine particles (Shapiro and Brenner, 1990). Granular and fibrous filters have been successfully used for remov- 
ing this mode of particle (Shapiro and Brenner, 1990; Bejan et al., 2004). The removal of particulates using filters is a long-established practice that is used in a variety of applications ranging from explosion vents in nuclear power facilities to vacuum cleaner exhausts (Bejan et al., 2004; Vafai and Giuliani, 1999); although existing models are still not suitable for prediction and design (Lehmann and Kasper, 2002).

Particle deposition in filters is an unsteady-state process, and the extent of deposition plays an important role in determining filter performance. Filter performance is monitored based on two quantities (Ghidaglia et al., 1991; Lehmann and Kasper, 2002; Bejan et al. 2004): a particle's penetration and pressure drop through the filter. Penetration or, alternatively, efficiency, which is a complementary parameter, measures the fraction of particles that cross the filter without being captured, therefore providing a measure of the ability of the structure to capture particles. The pressure drop indicates not only the influence of the solid matrix of the filter on the airflow, but also the influence of the particle's cake (i.e., the influence of the particle's cake on the filter's permeability to airflow). Generally, the pressure drop is used to measure the degree of a filter's clogging.

A good filter is characterized by very low penetration, while having high permeability (low pressure drop) to fluid flow. For a long time, the only quality criterion found in the literature, and used in the filter industry, was filter performance (or filter quality), defined as the ratio of the negative logarithm of penetration to the pressure drop across the filter (Bejan et al., 2004; Miguel 2004, Lehmann, and Kasper, 2002). The figure of merit resulting from this definition has the advantage that it can be calculated directly from parameters that can be measured easily. Its drawback is that filter performance is not a dimensionless quantity (it has the dimension of pressure). Therefore, its magnitude depends on the system used, and filters must be compared for specified filtration velocity, particle diameter, and particle loading. In order to avoid this, an alternative definition was put forward recently in which filter performance is defined as the product of the number of particles caught per unit of filter area and filter permeability (Miguel, 2003, 2004; Bejan et al., 2004),

During the past few decades, great advances have been made in the understanding of the mechanisms behind deposition of particulate suspensions. Regarding monitoring of particulate matter filtration, the majority of the theoretical work has been limited to the first moments of the deposition process, i.e., the filter is in almost its clean state (Lehmann and Kasper 2002; Bejan et al., 2004). However, during most of a filter's operation life, it runs partly loaded with particulate matter. As a result, during loading, the ability of the filter to hold particles together with the pressure drop through the filter tends to change substantially. This behavior has been demonstrated experimentally by several authors (e.g., Bejan et al., 2004). While the increase in the ability to hold particles is seen as a benefit, and even counted on to meet specifications, the augment of the pressure drop is undesirable. Therefore, dynamic modeling of filtration process is essential to rational design, optimization, and innovation of a filtration process.

Most of the work carried out on the prediction of a filtration process is based on the so-called single-fiber approach (e.g., Bergmann et al., 1978; Rembor et al., 1999; Bejan et al., 2004), or it is based on experimental correlations (see, e.g., Lehmann and Kasper, 2002; Jung and Tien, 1991; Stenhouse et al., 1992). Also, Shapiro and Brenner (1990), based on the idea that the filtration process is a convective-dispersive phenomenon, provided a theoretical definition for the filtration length and defined a filter's penetration in terms of the Peclet number. Quintard and Whitaker (1995), based on the same idea and applying the method of volume averaging, provided a description of a filters' efficiency.

In this paper, we consider the transport of fine particles through porous filters as a convective-dispersive phenomenon (see, for example, Bejan et al., 2004; Shapiro and Brenner, 1990; Quintard and Whitaker, 
1995) and develop transient comprehensive models for particle deposition within the filter, pressure drop through the filter and filter's performance.

\section{TRANSPORT AND DEPOSITION OF FINE PARTICLES IN POROUS MEDIA}

\subsection{Deposition Rate of Particles in a Deep Filter}

Consider a filter through which an airstream with suspension of fine particles is flowing (Fig. 1). As the airstream flows through the filter, some of the suspended particulate matter is deposited onto the solid matrix (e.g., beds, fibers). The rate of particle deposition is given by (Elimelech et al., 1995)

$$
\frac{\partial \Phi}{\partial t}=\Lambda u C
$$

where $\mathrm{u}$ represents the average fluid velocity within the filter, $\mathrm{C}$ is the particle mass concentration in the airstream within the filter, $t$ is the filtration time, and $\Lambda$ is the filtration coefficient.

The determination of the coefficient $\Lambda$ is rater complex. However, there is a related quantity, called the particulate matter transfer coefficient $(\Theta)$, which

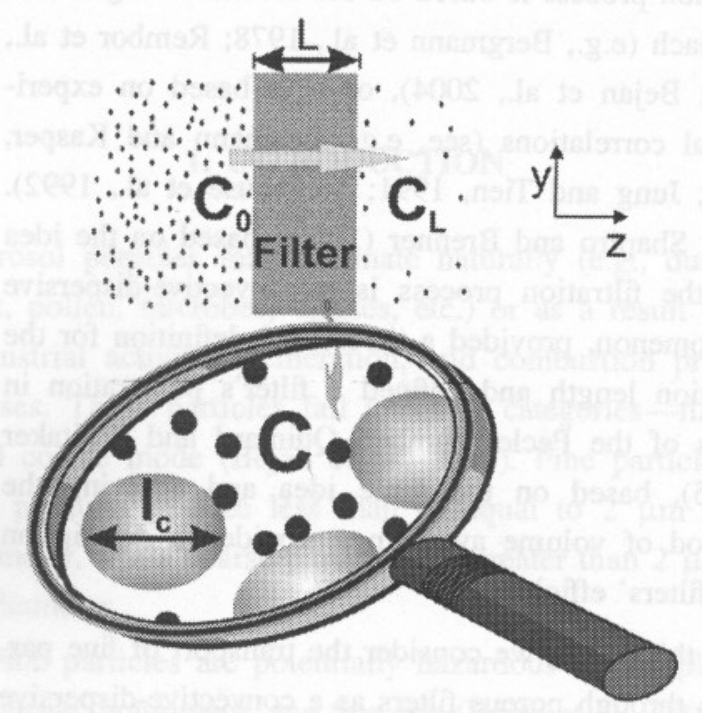

Figure 1. Schematic representation of the filtration process through a porous filter has been frequently used in the literature instead of the filter coefficient. The coefficients $\Lambda$ and $\Theta$ are local variables. However, in most cases, these two variables are given as averaged values over the filter medium thickness. The relation between $\Lambda$ and $\Theta$ is (Appendix A)

$$
\Lambda=\frac{\vartheta \Theta}{u}
$$

where $\vartheta$ represents the specific surface area of the filter.

Spielman and Friedlander (1984) presented comprehensive approaches that allow prediction of the particulate matter transfer coefficient as a function of the Peclet number, flow field, and particles-collector interaction for both fibrous and packed-bed filters. Another option is to obtain the particulate matter transfer coefficient experimentally based on the approaches reported in Appendix B.

The following variables are defined to make Eq. (1) dimensionless:

$$
\begin{aligned}
& \mathrm{Sh}=\frac{l_{c} \Theta}{D}, t^{*}=\frac{D t}{L^{2}}, \quad C^{*}=\frac{C}{\rho_{a i r}} \\
& \Phi^{*}=\frac{\Phi}{\rho_{\text {air }}}, \quad l_{c}^{*}=\frac{l_{c}}{L}, \quad \vartheta^{*}=\vartheta L
\end{aligned}
$$

where $D$ is the particle diffusion coefficient given by

$$
D=\frac{c_{u} k_{B} T}{6 \pi \mu r_{p}}
$$

and $\mathrm{Sh}$ is the Sherwood number, which represents the ratio of actual particulate mass transfer by a moving airstream to the particulate mass transfer that would occur by diffusion, $l_{c}$ is the characteristic length of the filter collector, $L$ is the thickness of the filter, $k_{B}$ is the Boltzmann's constant, $T$ is the absolute temperature, $\mu$ is the dynamic viscosity, $r_{p}$ is the particle radius, and $c_{c}$ is the Cunningham slip correction factor. Therefore, the dimensionless form of Eq. (1) reads as 


$$
\frac{\partial \Phi^{*}}{\partial t^{*}}=\operatorname{Sh} \frac{\vartheta^{*} C^{*}}{l_{c}^{*}}
$$

During the early moments of filtration, particles deposit directly onto the filter collectors (e.g., beds, fibers). After some time, particles start depositing directly onto the previously deposited particles (Tanthapanichakoon et al., 2003). Thus, the particulate matter transfer coefficient $\Theta$ reads as

$$
\Theta=(1-\varepsilon) \Theta_{c}+\varepsilon \Theta_{p}
$$

While the rate of particle deposition amount within the filter is given by

$$
\frac{\partial \Phi^{*}}{\partial t^{*}}=\frac{\partial \Phi_{c}^{*}}{\partial t^{*}}+\frac{\partial \Phi_{p}^{*}}{\partial t^{*}}=(1-\varepsilon) \operatorname{Sh}_{c} \frac{\vartheta^{*} C^{*}}{l_{c}^{*}}+\varepsilon \operatorname{Sh}_{p} \frac{\vartheta^{*} C^{*}}{l_{c}^{*}}
$$

where $\varepsilon$ is the fraction of collectors (e.g., beds, fibers) covered with particles; $\Phi_{c}^{*}$ is the particle deposition onto the filter collectors; $\Theta_{c}$ is the mass transfer coefficient, which accounts for deposition of particles on filter collectors; $\mathrm{Sh}_{c}$ is the Sherwood number, which accounts for deposition of particles on filter collectors; $\Phi_{p}^{*}$ is the particle deposition onto previously deposited particles; $\Theta_{p}$ is the mass transfer coefficient that accounts for deposition of particles on the previously deposited particles; and $\mathrm{Sh}_{p}$ is the Sherwood number, which accounts for deposition of particles on previously deposited particles.

The fraction of filter collectors that are covered with a layer of spherical particles is given by

$$
\varepsilon=\frac{\Phi_{c}^{*}}{C^{*}} \frac{k_{e}}{\alpha} \frac{\left(A_{p} / V_{p}\right)}{\left(A_{c} / V_{c}\right)}=\frac{\Phi_{c}^{*}}{C^{*}} \frac{k_{e}}{\alpha I_{p c}}
$$

Therefore, the rate of particle deposition onto the filter collectors and onto previously deposited particles can be rewritten as

$$
\frac{\partial \Phi_{c}^{*}}{\partial t^{*}}=\frac{\operatorname{Sh}_{c} \vartheta^{*}}{l_{c}^{*}} C^{*}-\frac{k_{e}}{\alpha I_{p c}} \frac{\operatorname{Sh}_{c} \vartheta^{*}}{l_{c}^{*}} \Phi_{c}^{*}
$$

$$
\frac{\partial \Phi_{p}^{*}}{\partial t^{*}}=\frac{k_{e}}{\alpha I_{p c}} \frac{\operatorname{Sh}_{p} \vartheta^{*}}{l_{c}^{*}} \Phi_{c}^{*}
$$

Here, $\mathrm{I}_{p c}$ is the aerosol particle interception parameter defined as $A_{p} V_{c} / A_{c} V_{p}, \Phi_{c}^{*}$ is the particle deposition amount deposited directly onto the filter's collector; $\Phi_{p}^{*}$ is the particle deposition onto previously deposited particles; $\alpha$ is the solidity of a new (unloaded) filter (i.e., the volume fraction of the solid matrix); $k_{e}$ is the excluded surface area factor, which has a theoretical minimum value close to 1.27 (Elimelech et al., 1995); $A_{p}$ is the particle surface area; $A_{c}$ is the filter collector surface area; $V_{p}$ is the particle volume; and $V_{c}$ is the filter collector volume.

Initial conditions are required to obtain solutions for Eqs. (9) and (10). A realistic scenario is to consider that the filter is completely free from particles (unloaded) at beginning of the filtration process, such that

$$
\Phi_{c}^{*}=\Phi_{p}^{*}=0 \text { at } t^{*}=0
$$

By considering the initial condition defined by Eq. (11) and constant filtration velocity, which is a realistic assumption for many applications (Bergman et al., 1978; Lehmann and Kasper, 2002), we obtain

$$
\begin{aligned}
& \Phi_{c}^{*}=\frac{\alpha I_{p c}}{k_{e}} C^{*}\left[1-\exp \left(-\frac{k_{e}}{\alpha I_{p c}} \frac{\mathrm{Sh}_{c} \vartheta^{*}}{l_{c}^{*}} t^{*}\right)\right] \\
& \Phi_{p}^{*}=\frac{\operatorname{Sh}_{p} \vartheta^{*}}{l_{c}^{*}} C^{*} t^{*}-\frac{\operatorname{Sh}_{p} \alpha I_{p c}}{\operatorname{Sh}_{c} k_{e}} C^{*} \\
& \times\left[1-\exp \left(-\frac{k_{e}}{\alpha I_{p c}} \frac{\operatorname{Sh}_{c} \vartheta^{*}}{l_{c}^{*}} t^{*}\right)\right]
\end{aligned}
$$

The total deposit amount inside the filter $\left(\Phi^{*}\right)$ is the sum of $\Phi_{c}^{*}$ and $\Phi_{p}^{*}$

$$
\begin{aligned}
& \Phi^{*}=\frac{\mathrm{Sh}_{p} \vartheta^{*}}{l_{c}^{*}} C^{*} t^{*}+\frac{\alpha I_{p c}}{k_{e}} C^{*}\left(1-\frac{\mathrm{Sh}_{p}}{\mathrm{Sh}_{c}}\right) \\
& \times\left[1-\exp \left(-\frac{k_{e}}{\alpha I_{p c}} \frac{\mathrm{Sh}_{c} \vartheta^{*}}{l_{c}^{*}} t^{*}\right)\right]
\end{aligned}
$$


In order to obtain the amount of particles deposited within the filter, the concentration of particles in the airstream $\left(C^{*}\right)$ is required. This topic is addressed in the next section.

\subsection{Macroscopic Description of Particulate Matter Flow with Deposition}

Filtration of fine particles represents an example of transport in porous media that can be analyzed based on the convective-dispersive phenomenon (see, e.g., Shapiro and Brenner, 1990; Quintard and Whitaker, 1995; Bejan et al., 2004). Let us consider onedimensional transport of particles through a filter medium (i.e., the particulate matter is considered to be both horizontally and vertically well mixed so that concentrations only vary along the flow). Under steady conditions, the concentration of dilute particulate matter $(C)$ flowing through the porous filter (Fig. 1) is governed by the macroscopic equation

$$
u \frac{\partial C_{z}}{\partial z}=D \frac{\partial^{2} C_{z}}{\partial z^{2}}+\frac{\partial \Phi}{\partial t}
$$

Here, $C_{z}$ is the concentration of particulate matter in the airstream and $\Phi$ represents the collection of particles by the filter (i.e., particles removed from the suspension), which for continuity reasons is $\Phi=$ $-\Phi_{c}-\Phi_{p}$.

By defining the two additional dimensionless variables

$$
\mathrm{Pe}=\frac{l_{c} u}{D}, z^{*}=\frac{z}{L}
$$

the dimensionless form of Eq. (15) reads

$$
\frac{\partial \Phi^{*}}{\partial t^{*}}=\frac{\partial^{2} C_{z}^{*}}{\partial z^{* 2}}-\frac{\mathrm{Pe}}{l_{c}^{*}} \frac{\partial C_{z}^{*}}{\partial z^{*}}
$$

where $\mathrm{Pe}$ is the Peclet number, a measure of relative importance of convection to diffusion. The higher the Peclet number, the more important is convection.
Taking into account Eqs. (14) and (17) and neglecting the rate of particle release as compared with the particle attachment rate, the concentration of particles flowing through the filter is described by

$$
\begin{aligned}
& -\frac{\partial^{2} C_{z}^{*}}{\partial z^{* 2}}+\frac{\operatorname{Pe}}{l_{c}^{*}} \frac{\partial C_{z}^{*}}{\partial z^{*}}+\frac{\vartheta^{*}}{l_{c}^{*}}\left[\mathrm{Sh}_{p}+\mathrm{Sh}_{c}\left(1-\frac{\mathrm{Sh}_{p}}{\mathrm{Sh}_{c}}\right)\right. \\
& \left.\times \exp \left(-\frac{k_{e}}{\alpha I_{p c}} \frac{\mathrm{Sh}_{c} \vartheta^{*}}{l_{c}^{*}} t^{*}\right)\right] C_{z}^{*}=0
\end{aligned}
$$

Boundary conditions are required for solving Eq. (18). A possible scenario is to fix the concentration of particles sources at the upstream boundary. This case is of great importance since it corresponds to many industrial pollution sources in which particles are released at a constant rate for some periods. The boundary conditions are

$$
\begin{aligned}
& C_{z}^{*}=C_{0}^{*} \text { at } z^{*}=0 \\
& \frac{\partial C_{z}^{*}}{\partial z^{*}}=0 \text { at } z^{*}=L^{*}
\end{aligned}
$$

where $C_{0}^{*}$ is the dimensionless upstream particle concentration.

The solution to Eq. (18) under the boundary conditions (19) and (20) reads as

$$
\begin{aligned}
& C_{z}^{*}=\frac{C_{0}^{*}}{1-\frac{b_{1}}{b_{2}} \exp \left[\left(b_{1}-b_{2}\right) L^{*}\right]} \\
& \times\left[\exp \left(b_{1} z^{*}\right)-\frac{b_{1}}{b_{2}} \exp \left[\left(b_{1}-b_{2}\right) L^{*}\right] \exp \left(b_{2} z^{*}\right)\right]
\end{aligned}
$$

with

$$
\begin{aligned}
& b_{1}=\frac{1}{2} \frac{\mathrm{Pe}}{l_{c}^{*}} \\
& +\sqrt{\frac{1}{4}\left(\frac{\mathrm{Pe}}{l_{c}^{*}}\right)^{2}+\frac{\vartheta^{*}}{l_{c}^{*}}\left[\mathrm{Sh}_{p}+\mathrm{Sh}_{c}\left(1-\frac{\mathrm{Sh}_{p}}{\mathrm{Sh}_{c}}\right) \exp \left(-\frac{k_{e}}{\alpha I_{p c}} \frac{\mathrm{Sh}_{c^{\vartheta *}}}{l_{c}^{*}} t^{*}\right)\right]} \\
& b_{2}=\frac{1}{2} \frac{\mathrm{Pe}}{l_{c}^{*}} \\
& -\sqrt{\left.\frac{1}{4}\left(\frac{\mathrm{Pe}}{l_{c}^{*}}\right)^{2}+\frac{\vartheta^{*}}{l_{c}^{*}} \operatorname{Sh}_{p}+\mathrm{Sh}_{c}\left(1-\frac{\mathrm{Sh}_{p}}{\mathrm{Sh}_{c}}\right) \exp \left(-\frac{k_{e}}{\alpha I_{p c}} \frac{\mathrm{Sh}_{\vartheta^{2}} \vartheta^{*}}{l_{c}^{*}} t^{*}\right)\right]}
\end{aligned}
$$


Additionally, the spatially averaged concentration is given by

$$
\begin{aligned}
& C^{*}=\frac{\int_{0}^{L^{*}} C_{z}^{*} d z^{*}}{\int_{0}^{L^{*}} d z^{*}}=\frac{C_{0}^{*}}{1-\frac{b_{1}}{b_{2}} \exp \left[\left(b_{1}-b_{2}\right) L^{*}\right]} \\
& \times\left[\frac{1}{b_{1}}\left[\exp \left(b_{1} L^{*}\right)-1\right]-\frac{b_{1}}{b_{2}^{2}}\left[\exp \left(b_{2} L^{*}\right)-1\right]\right. \\
& \left.\times \exp \left[\left(b_{1}-b_{2}\right) L^{*}\right]\right]
\end{aligned}
$$

Equations (21) and (24) describe the spatial distribution and the average concentration of particles in the airstream within the filter, respectively.

\section{DEPOSITION OF AEROSOLS WITHIN THE FILTER}

Based on the formulation developed in the previous sections, we are able to obtain the amount of particles deposited within the filter. By combining Eqs. (12)(14) with Eq. (24), particle deposition onto the filter's collectors is given by

$$
\begin{aligned}
& \Phi_{c}^{*}=\frac{C_{0}^{*}}{1-\frac{b_{1}}{b_{2}} \exp \left[\left(b_{1}-b_{2}\right) L^{*}\right]}\left[\frac{1}{b_{1}}\left[\exp \left(b_{1} L^{*}\right)-1\right]\right. \\
& \left.-\frac{b_{1}}{b_{2}^{2}}\left[\exp \left(b_{2} L^{*}\right)-1\right] \exp \left[\left(b_{1}-b_{2}\right) L^{*}\right]\right] \\
& \times\left\{\frac{\alpha I_{p c}}{k_{e}}\left[1-\exp \left(-\frac{k_{e}}{\alpha I_{p c}} \frac{\operatorname{Sh}_{c} \vartheta^{*}}{l_{c}^{*}} t^{*}\right)\right]\right\}
\end{aligned}
$$

while the particle deposition onto previously deposited particles reads as

$$
\begin{aligned}
& \Phi_{p}^{*}=\frac{C_{0}^{*}}{1-\frac{b_{1}}{b_{2}} \exp \left[\left(b_{1}-b_{2}\right) L^{*}\right]}\left[\frac{1}{b_{1}}\left[\exp \left(b_{1} L^{*}\right)-1\right]\right. \\
& \left.-\frac{b_{1}}{b_{2}^{2}}\left[\exp \left(b_{2} L^{*}\right)-1\right] \exp \left[\left(b_{1}-b_{2}\right) L^{*}\right]\right] \\
& \times\left\{\frac{\operatorname{Sh}_{p} \vartheta^{*}}{l_{c}^{*}} t^{*}-\frac{\operatorname{Sh}_{p} \alpha I_{p c}}{\operatorname{Sh}_{c} k_{e}}\left[1-\exp \left(-\frac{k_{e}}{\alpha I_{p c}} \frac{\operatorname{Sh}_{c} \vartheta^{*}}{l_{c}^{*}} t^{*}\right)\right]\right\}
\end{aligned}
$$

Therefore, the total particle deposition within the filter is given by

$$
\begin{aligned}
& \Phi^{*}=\frac{C_{0}^{*}}{1-\frac{b_{1}}{b_{2}} \exp \left[\left(b_{1}-b_{2}\right) L^{*}\right]} \\
& \times\left[\frac{1}{b_{1}}\left[\exp \left(b_{1} L^{*}\right)-1\right]-\frac{b_{1}}{b_{2}^{2}}\left[\exp \left(b_{2} L^{*}\right)-1\right] \exp \left[\left(b_{1}-b_{2}\right) L^{*}\right]\right] \\
& \times\left\{\frac{\mathrm{Sh}_{p} \vartheta^{*}}{l_{c}^{*}} t^{*}+\frac{\alpha I_{p c}}{k_{e}}\left(1-\frac{\mathrm{Sh}_{p}}{\mathrm{Sh}_{c}}\right)\left[1-\exp \left(-\frac{k_{e}}{\alpha I_{p c}} \frac{\mathrm{Sh}_{c} \vartheta^{*}}{l_{c}^{*}} t^{*}\right)\right]\right\}
\end{aligned}
$$

where $b_{1}$ and $b_{2}$ are given by Eqs. (22) and (23).

Figure 2 shows the effect of the dimensionless numbers $\left(\mathrm{Pe}, \mathrm{Sh}_{c}, \mathrm{Sh}_{p}\right)$ and geometrical parameter $\left(I_{p c}\right)$ on $\Phi^{*} / C_{0}^{*}$. The plot shows that the amount of particles deposited increases with the Peclet number, the effect being stronger in the range of Pe between 0.1 and 50. Results also indicate that during the early stages of the filtration process, deposition is mainly influenced by $\mathrm{Sh}_{c}$, but at later stages $\mathrm{Sh}_{p}$ becomes more important. Therefore, during the early stages deposition is favored when $\mathrm{Sh}_{c}$ is larger than $\mathrm{Sh}_{p}$, but later on deposition is enhanced when $\mathrm{Sh}_{p}$ is larger than $\mathrm{Sh}_{c}$. Figure 2 also reveals that $\Phi^{*} / C_{0}^{*}$ is influenced by $I_{p c}$; however, this influence negligible when the filter approach clogging. Notice that when $\mathrm{Sh}_{c}$ is larger than $\mathrm{Sh}_{p}$, the increase of the interception parameter favors deposition. The opposite effect occurs when $\mathrm{Sh}_{c}$ is smaller than $\mathrm{Sh}_{p}$.

The time evolution of $\Phi_{c}^{*} / C^{*}, \Phi_{p}^{*} / C^{*}$, and $\Phi^{*} / C^{*}$ is presented in Fig. 3. As expected, the plot shows that during the early stages of filtration, the main contribution for the deposition is the fraction of particles deposited onto the filter's collector $\left(\Phi_{c}^{*}\right)$. After $t^{*}=t_{c c}^{*} \sim 0.62$, the fibers or the beds that constitute the filter become completely covered with particles. Therefore, particles cannot deposit directly onto the filter's collectors (e.g., fibers, beds) and have to deposit on particles already attached to these collectors. Filtration becomes exclusively due to particles that deposit directly onto previously deposited particles $\left(\Phi_{p}^{*}\right)$. This result is in agreement with the findings obtained experimentally by other authors (Lehmann and Kasper, 2002; Tanthapanichakoon et al., 2003).

According to Eq. (8), $t_{c c}^{*}$ is reached when

$$
\left(\Phi^{*}\right)_{t_{c c}^{*}}=\frac{\alpha I_{p c}}{k_{e}} C^{*}
$$


a)
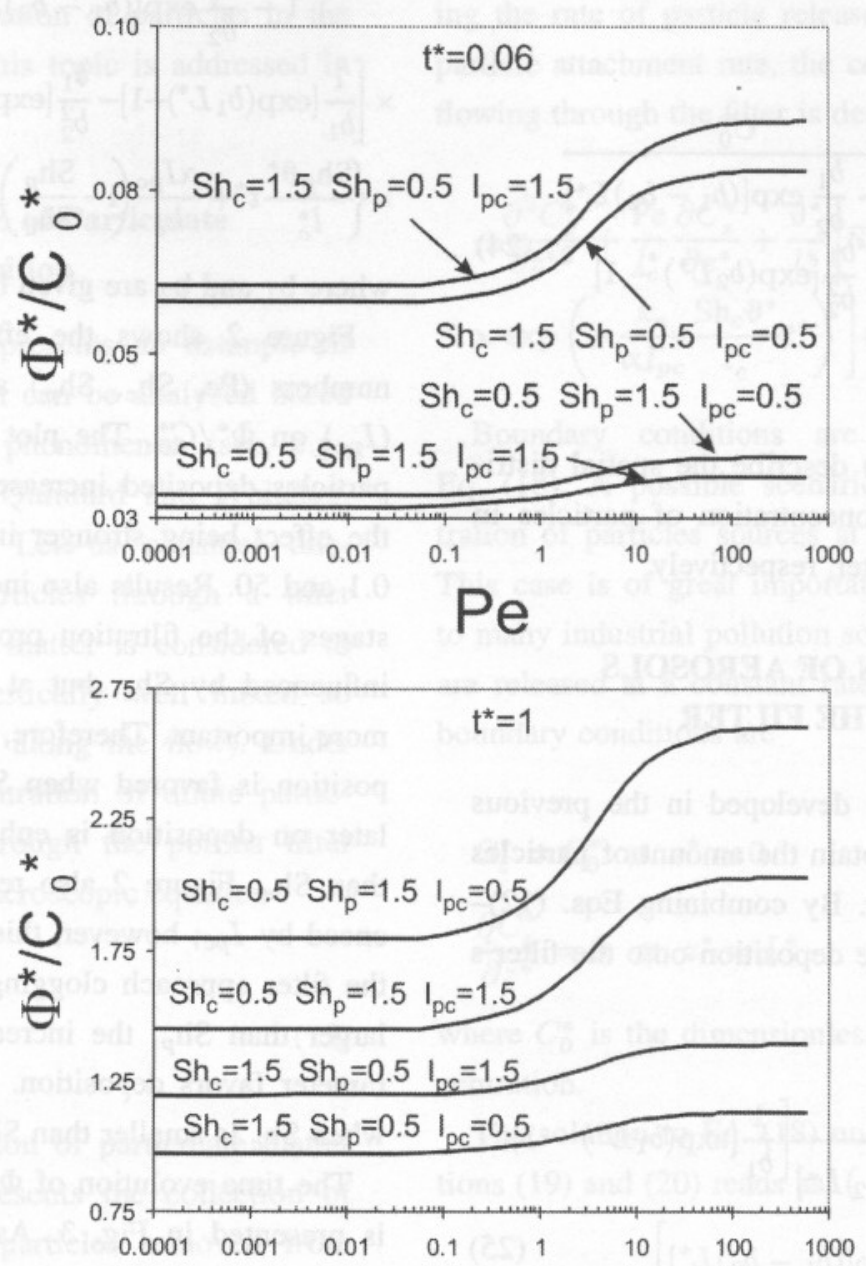

b)
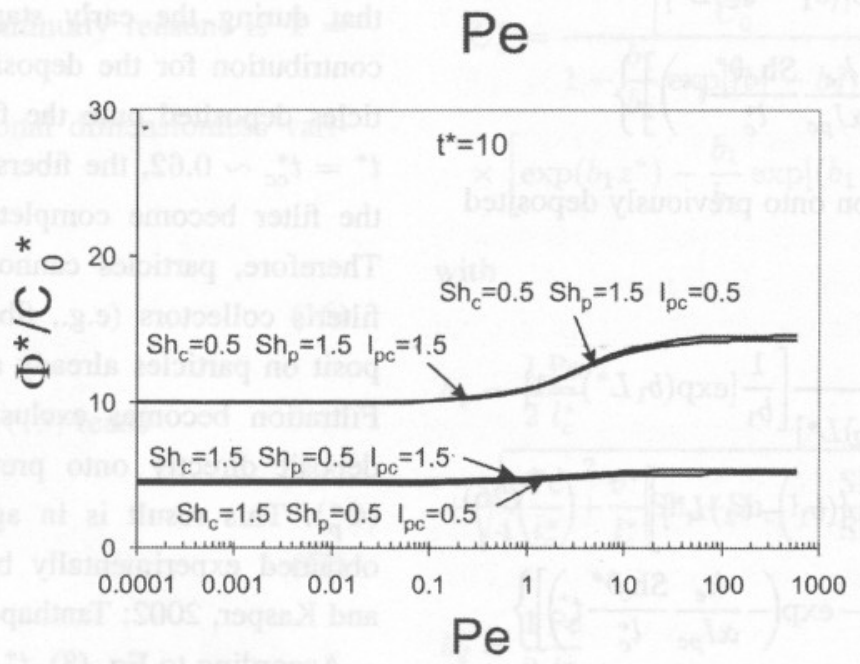

c)

Figure 2. Effects of Peclet number, Sherwood number, and interception parameter on $\Phi^{*} / C_{0}^{*}$ : a) $t^{*}=0.06, \alpha=0.5$, $k_{e}=1.27$; b) $t^{*}=1, \alpha=0.5, k_{e}=1.27$; c) $t^{*}=10, \alpha=0.5, k_{e}=1.27$ 


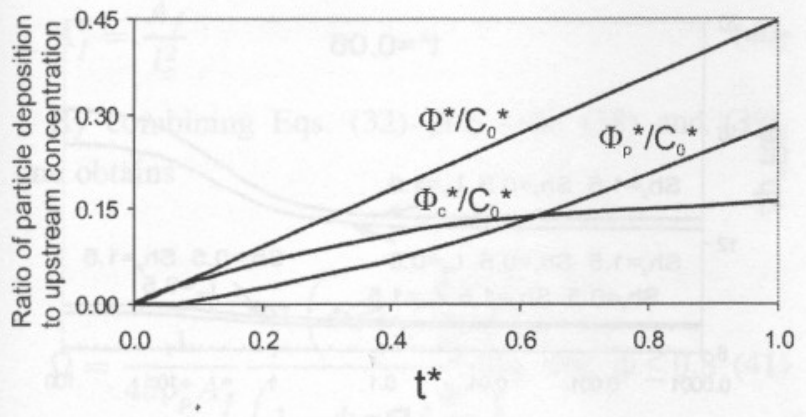

Figure 3. Time evolution of $\Phi_{c}^{*} / C^{*}, \Phi_{p}^{*} / C^{*}$, and $\Phi^{*} / C^{*}$ $\left(\alpha=0.5, k_{e}=1.27\right)$

The time $t_{\text {life }}^{*}$ corresponds to filter clogging. If $\phi$ is the void fraction of the filter (i.e., the porosity), the lifetime of the filter is reached for

$$
\left(\Phi^{*}\right)_{t_{i i f e}^{*}}=\phi \zeta \rho_{p}^{*}
$$

where $\zeta$ is the ratio between filter volume and particle volume, and $\rho_{p}^{*}$ is the dimensionless particle density $\left(\rho_{p} / \rho_{\text {air }}\right)$. Therefore, Eqs. (28) and (29) may constitute the criteria to determine $t_{c c}^{*}$ and $t_{\text {life }}^{*}$.

\section{PRESSURE DROP THROUGH THE FILTER}

It is well known that as the filter becomes clogged, the pressure drop through the filter increases significantly (see, e.g., Bejan et al., 2004). The classical approach for filtration analysis at low Reynolds number $(\operatorname{Re}<1)$ is based on Darcy's law, which in dimensionless form reads (see, e.g., Miguel 2003) as

$$
\Delta p^{*}=\frac{L^{*}}{K^{*} R e}
$$

with

$$
p^{*}=\frac{p}{\rho u^{2}} ; \quad K^{*}=\frac{K}{l_{c}^{2}} ; \quad R e=\frac{\rho u l_{c}}{\mu}
$$

Here, $p$ is pressure, $K$ is filter permeability, $\mu$ is dynamic fluid velocity, and $\operatorname{Re}$ is the Reynolds number. The permeability of filters with porosities $(\phi)$ up to 0.8 can be obtained from the hydraulic radius theory of Carman-Kozeny (Nield and Bejan, 1999; Bejan et al., 2004) as

$$
K^{*}=\frac{1}{45} \frac{\phi^{3}}{(1-\phi)^{2}}
$$

or alternatively, for permeabilities higher than 0.8 , by (Koponen et al., 1998; Pinela et al., 2005)

$$
K^{*}=\frac{5.56}{\exp [10.1(1-\phi)]-1}
$$

Particle deposition within the filter originates a decrease in the filter's porosity. This change in porosity can be related to the amount of particles deposited in the filter according to (Miguel 2003, 2004)

$$
\phi=\phi_{0}-\zeta \frac{\Phi^{*}}{\rho_{p}^{*}}
$$

where $\phi_{0}$ is the porosity of a new (unloaded) filter and $\zeta$ is the ratio of filter volume to particle volume.

By combining Eqs. (27) and (32)-(34) together with Eq. (30), the pressure drop reads as

$$
\begin{aligned}
& \Delta p^{*}=45 \frac{L^{*}}{\operatorname{Re}} \frac{(1-\varphi)^{2}}{\varphi^{3}} \text { for } \phi \leq 0.8 \\
& \Delta p^{*}=\frac{L^{*}\{\exp [10.1(1-\varphi)]-1\}}{5.56 \operatorname{Re}} \text { for } 0.8<\phi<1
\end{aligned}
$$

with

$$
\varphi=\phi_{0}-\frac{\zeta C_{0}^{*}}{\rho_{p}^{*}-\rho_{p}^{*} \frac{b_{1}}{b_{2}} \exp \left[\left(b_{1}-b_{2}\right) L^{*}\right]}
$$

$\times\left[\frac{1}{b_{1}}\left[\exp \left(b_{1} L^{*}\right)-1\right]-\frac{b_{1}}{b_{2}^{2}}\left[\exp \left(b_{2} L^{*}\right)-1\right] \exp \left[\left(b_{1}-b_{2}\right) L^{*}\right]\right]$ $\times\left\{\frac{\mathrm{Sh}_{p} \vartheta^{*}}{l_{c}^{*}} t^{*}+\frac{\alpha I_{p c}}{k_{e}}\left(1-\frac{\mathrm{Sh}_{p}}{\mathrm{Sh}_{c}}\right)\left[1-\exp \left(-\frac{k_{e}}{\alpha I_{p c}} \frac{\mathrm{Sh}_{c} \vartheta^{*}}{l_{c}^{*}} t^{*}\right)\right]\right\}$

The curve representing the variation of $\Delta p^{*} \operatorname{Re}$ with time is represented in Fig. 4. Notice that the 


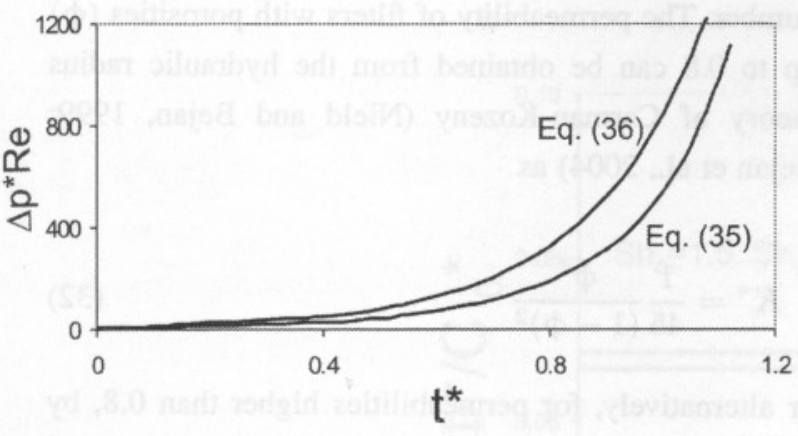

Figure 4. Time evolution of $\Delta p^{*} \operatorname{Re}\left(k_{e}=1.27, \mathrm{Pe}=\right.$ $\mathrm{Sh}_{c}=\mathrm{Sh}_{p}=I_{p c}=0.5$ )

plot displays three different regions: an initial small increase, followed by a transition region, and a final region of rapid increase that corresponds to a filter close to clogging. This profile of variation agrees with experimental results presented by Callé et al. (2002) and Miguel (2003).

Figure 5 shows $\Delta p^{*} \operatorname{Re}$ versus the Peclet number for various Sherwood numbers and interception factors. The plot reveals an increase of $\Delta p^{*} \operatorname{Re}$ with the Peclet number, and this tendency is more noteworthy between 0.1 and 50 . Initially, $\Delta p^{*} \mathrm{Re}$ is enhanced if $\mathrm{Sh}_{c}$ is larger than $\mathrm{Sh}_{p}$, but at later stages the opposite occurs (i.e., $\Delta p^{*} \mathrm{Re}$ is enhanced when $\mathrm{Sh}_{p}$ is larger than $\mathrm{Sh}_{c}$ ). This result stresses the importance of both $\mathrm{Sh}_{c}$ and $\mathrm{Sh}_{p}$ in the filtration process. In addition, when $\mathrm{Sh}_{c}$ is larger than $\mathrm{Sh}_{p}$, an increase of the interception parameter favors pressure drop. The opposite effect occurs when $\mathrm{Sh}_{c}$ is smaller than $\mathrm{Sh}_{p}$.

Notice also that the tendency of variation in $\Delta p^{*} R e$ with the Peclet and Sherwood numbers, as well as the interception factor, is similar to that obtained in Fig. 2 for particle deposition. This is an expected result because the pressure drop through the filter is strongly dependent on the amount of particles deposited.

\section{FILTER PERFORMANCE}

A high-quality air filter is characterized by high collection efficiency of particles associated with high per-
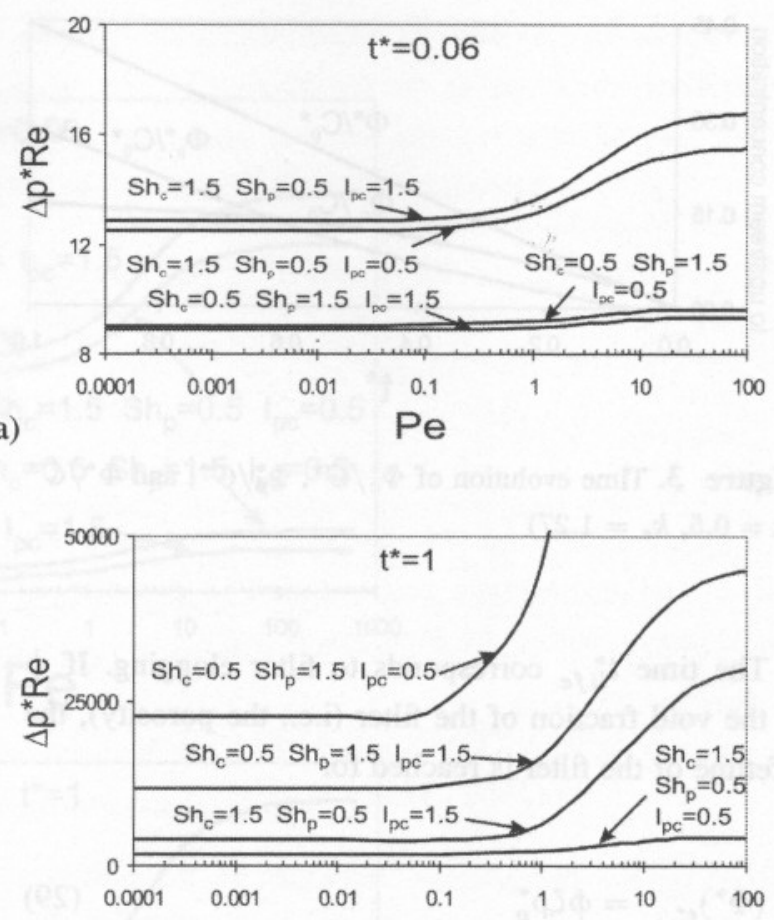

b)

$\mathrm{Pe}$

Figure 5. Effects of Peclet number, Sherwood number, and interception parameter on $\Delta p^{*} \operatorname{Re}:$ a) $t^{*}=0.06$, $\left.k_{e}=1.27 ; \mathbf{b}\right) t^{*}=1, k_{e}=1.27$

meability. Filter performance can be evaluated from the product of the number of particles caught per unit of filter area and the filter permeability (Miguel, 2003;

Bejan et al., 2004) as

$$
\Omega=\frac{\Phi}{\rho_{p} A_{f}} K
$$

Here, $\Omega$ is the filter performance or filter quality. For convenience, in terms of dimensionless quantities, it reads as

$$
\Omega=\frac{\Phi^{*}}{\rho_{p}^{*} A_{f}^{*}} K^{*}
$$

with 


$$
A_{f}^{*}=\frac{A_{f}}{l_{c}^{2}}
$$

By combining Eqs. (32)-(36) with (38) and (39), one obtains

$$
\begin{aligned}
& \Omega=\frac{1}{45 \rho_{p}^{*} A_{f}^{*}} \frac{\Phi^{*}\left(\phi_{0}-\zeta \frac{\Phi^{*}}{\rho_{p}^{*}}\right)^{3}}{\left(1-\phi_{0}+\zeta \frac{\Phi^{*}}{\rho_{p}^{*}}\right)^{2}} \text { for } \phi \leq 0.8 \\
& \Omega=\frac{5.56}{\rho_{p}^{*} A_{f}^{*}} \\
& \times \frac{\Phi^{*}}{\left\{\exp \left[10.1\left(1-\phi_{0}+\zeta \frac{\Phi^{*}}{\rho_{p}^{*}}\right)\right]-1\right\}} \text { for } 0.8<\phi<1(
\end{aligned}
$$

where $\Phi^{*}$ is obtained from Eq. (27).

The time variation of performance is shown in Figs. 6-8. These plots display four different regions: an initial steep increase until it reaches a maximum (the peak), followed by a steep decrease, a transition range, and an ending range of constant performance. This last range corresponds to a filter close to clogging.

Figure 6 shows that the Peclet number has different effects on performance before and after reaching the peak. Before the peak, performance increases with the Peclet number. On the other hand, after the peak high Peclet numbers lower performance. Also, the

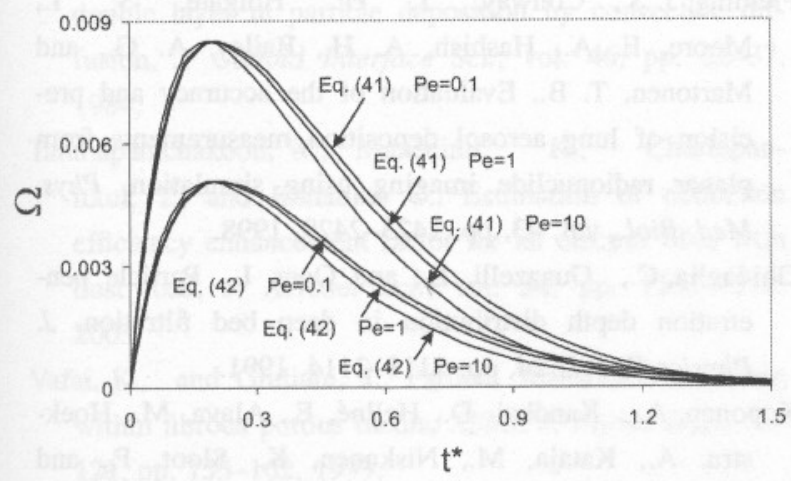

Figure 6. Filter performance versus time for various Peclet numbers $\left(k_{e}=1.27\right)$ maximum performance is reached earlier for high Peclet numbers [i.e., for $\mathrm{Pe}=0.1$, the peak is reached at $t^{*}=0.21$ (Eq. 41) and 0.22 (Eq. 42), while for $\mathrm{Pe}=10$, it is reached at $\mathrm{t}^{*}=0.18$ (Eq. 41) and 0.19 (Eq. 42)]. This means that the time required for replacing a filter decreases with the Peclet number.

The effects of the Sherwood number and the interception factor are shown in Figs. 7 and 8. These plots reveal that the maximum performance (the peak) occurs earlier when $\mathrm{Sh}_{c}$ is larger than $\mathrm{Sh}_{p}$. These figures also show that as the interception factor in-

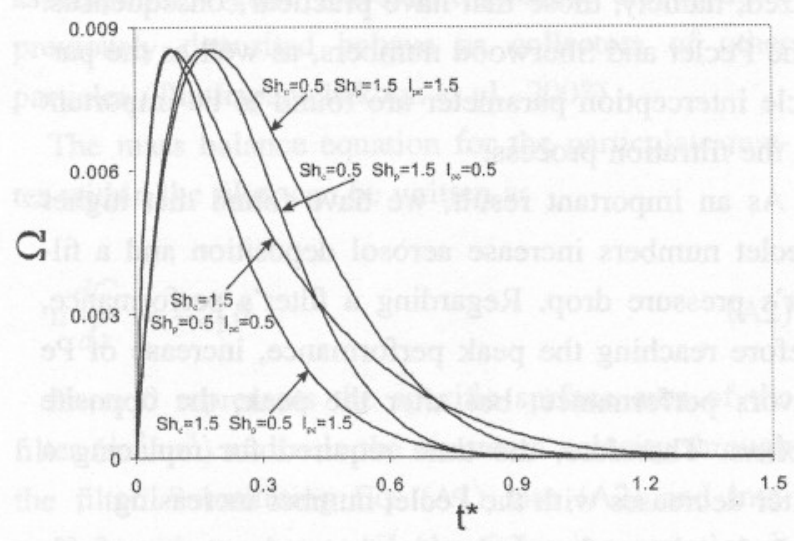

Figure 7. Effects of Sherwood number and interception parameter on filter performance [Eq. (41)] $\left(\alpha=0.5, k_{e}=\right.$ 1.27)

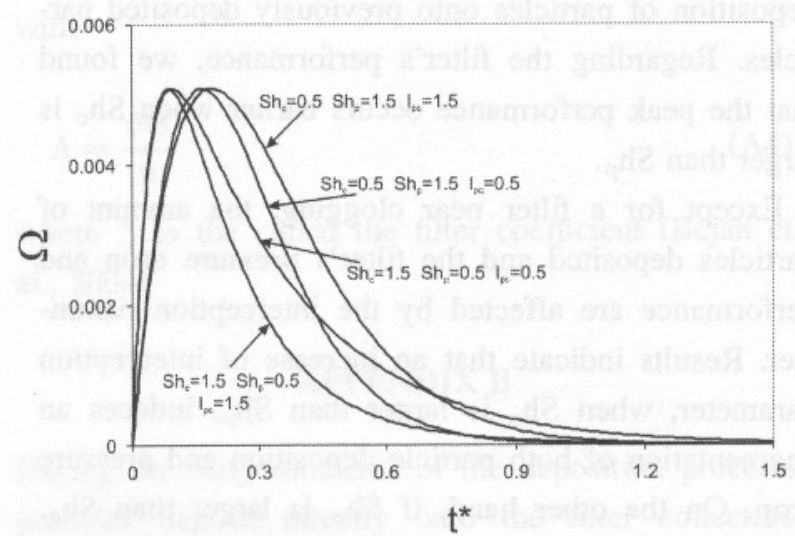

Figure 8. Effects of Sherwood number and interception parameter on filter performance [(Eq. (42)] $(\alpha=0.1$, $\left.k_{e}=1.27\right)$ 
creases, maximum performance occurs later when $\mathrm{Sh}_{c}$ is smaller than $\mathrm{Sh}_{p}$, while it occurs earlier when $\mathrm{Sh}_{c}$ is larger than $\mathrm{Sh}_{p}$.

\section{CONCLUDING REMARKS}

Transient analytical models for predicting the deposition of aerosol particles, pressure drop through filters, and a filter's performance were derived by considering that fine mode particles' transport through porous filters is a convective-dispersive phenomenon. Based on these approaches, some important features were analyzed, namely, those that have practical consequences. The Peclet and Sherwood numbers, as well as the particle interception parameter are found to be important in the filtration process.

As an important result, we have found that higher Peclet numbers increase aerosol deposition and a filter's pressure drop. Regarding a filter's performance, before reaching the peak performance, increase of Pe favors performance; but after the peak, the opposite occurs. Therefore, the time required for replacing a filter decreases with the Peclet number increasing.

It has been found that in the early stages of filtration, particle deposition and a filter's pressure drop increase with the Sherwood number that accounts for deposition of particles onto filter collectors, while at later stages by the Sherwood number accounting for deposition of particles onto previously deposited particles. Regarding the filter's performance, we found that the peak performance occurs earlier when $\mathrm{Sh}_{c}$ is larger than $\mathrm{Sh}_{p}$.

Except for a filter near clogging, the amount of particles deposited and the filter's pressure drop and performance are affected by the interception parameter. Results indicate that an increase of interception parameter, when $\mathrm{Sh}_{c}$ is larger than $\mathrm{Sh}_{p}$, induces an augmentation of both particle deposition and pressure drop. On the other hand, if $\mathrm{Sh}_{p}$ is larger than $\mathrm{Sh}_{c}$, the interception parameter has an opposite effect on deposition and pressure drop.

The models presented in this study may contribute to the comprehension of the filtration of fine mode particles, as well as for the improvement of filter design.

\section{ACKNOWLEDGMENT}

This work was supported by the Foundation for Science and Technology (FCT) under project POCTI/EME/59909/2004.

\section{REFERENCES}

Bejan, A., Dincer, I., Lorente, S., Miguel, A. F., and Reis, A. H., Porous and Complex Flow Structures in Modern Technologies, Springer-Verlag, New York, 2004.

Bergman, W., Taylor, R. D., Miller, H. H., Biermann, A. H., Hebard, H. D., da Roza, R. A., and Lum, B. Y., Enhanced filtration program at LLLa progress report, 15th DOE Nuclear Air Cleaning Conference, New York, First, M., ed., pp. 1058-1097, 1978.

Callé, S., Thomas, D., Contal, P., Appert-Collin, J. C., and Bémer, D., Effect of particle size on filter pressure drop and its modeling, Particle Loading and Kinetics of Filtration in Fibrous Filters, Institut für Mechanische Verfahrenstechnik und Mechanik, Universität Karlsruhe, Germany, 2002.

Elimelech, M., Gregory, J., Jia, X., and Williams, R. A., Particle Deposition and Aggregation: Measurements, Modelling and Simulation, Butterworth-Heinemann, Oxford, 1995.

Fleming, J. S., Conway, J. H., Holgate, S. T., Moore, E. A., Hashish, A. H., Bailey, A. G., and Martonen, T. B., Evaluation of the accuracy and precision of lung aerosol deposition measurements from planar radionuclide imaging using simulation, Phys. Med. Biol., vol. 43, pp. 2423-2429, 1998.

Ghidaglia, C., Guazzelli, E., and Oger, L., Particle penetration depth distribution in deep bed filtration, $J$. Physics D, vol. 24, pp. 2111-2114, 1991.

Koponen, A., Kandhai, D., Hellné, E., Alava, M., Hoekstra, A., Kataja, M., Niskanen, K., Sloot, P., and Timonen, J., Permeability of three-dimensional random fiber web, Phys. Rev. Lett., 80, pp. 716-719, 1998.

Lehmann, M. J., and Kasper, G., eds., Particle Loading and Kinetics of Filtration in Fibrous Filters, Insti- 
tut für Mechanische Verfahrenstechnik und Mechanik, Universität Karlsruhe, Germany, 2002.

Miguel, A. F., Effect of air humidity on the evolution of permeability and performance of a fibrous filter during loading with hygroscopic and non-hygroscopic particles, J. Aerosol Sci., vol. 34, pp. 783-799, 2003.

Miguel, A. F., Porous Media and Filtration, Emerging Technologies and Techniques in Porous Media, Kluwer, Dordrecht, pp. 419-431, 2004.

Pinela, J., Kruz, S., Miguel, A. F., Reis, A. H., and Aydin M., Permeability-porosity relationship assessment by 2-D numerical simulations, Proc. of International Symposium on Transport Phenomena (ISTP-16), Prague, Czech Republic, pp. 159-164, 2005.

Quintard, M., and Whitaker, S., Aerosol filtration: an analysis using the method of averaging volume, $J$. Aerosol Sci., vol. 26, pp. 1227-1255, 1995.

Reis, A. H., Miguel, A. F., and Aydin, M., Constructal theory of flow architecture of the lungs, Med. Phys., 31, pp. 1135-1140, 2004.

Rembor, H. J., Maus, R., and Umhauer, H., Measurement of single fibre efficiencies at critical values of the Stokes number, Part. Sys. Charact., vol. 16, 54-59, 1999.

Schwartz, J., and Dockery, D. W., Increased mortality in Philadelphia associated with daily air pollution concentration, Am. Rev. Resp. Dis., vol. 145, pp. 600-604, 1992.

Shapiro, M., and Brenner, H., Dispersion/reaction model of aerosol collection by porous filters, J. Aerosol Sci., vol. 21, pp. 97-125, 1990.

Spielman, L. A., and Friedlander, S., Role of electrical double layer in particle deposition by convective diffusion, J. Colloid Interface Sci., vol. 46, pp. 22-37, 1984.

Tanthapanichakoon, W., Maneeintr, K., Charinpanitkul, T., and Kanaoka, C., Estimation of collection efficiency enhancement factor for an electret fiber with dust load, J. Aerosol Sci., vol. 34, pp. 1505-1522, 2003.

Vafai, K., and Giuliani, J., Particle Arrestance modeling within fibrous porous media, ASME J. Fluids Eng., vol. 121, pp. 155-162, 1999.

US EPA 1997 Health and Environmental Effects of Particulate Matter, Office of Air Quality Planning and Standards, July 1997.

\section{APPENDIX A}

The flux of particulate matter $\left(J_{p}\right)$ onto a filter collector (e.g., fibers, beds) is given by

$$
J_{p}=\Theta\left(C-C_{c}\right)
$$

where $\Theta$ is the particulate matter transfer coefficient, $C$ the bulk particle concentration, and $C_{c}$ the particle concentration on the filter's collectors' surfaces. The term $C_{c}$ can be considered null because we assume that the collectors are perfect sinks of particles. This assumption is quite reasonable because the particles previously deposited behave as collectors of other particles (Tanthapanichakoon et al., 2003).

The mass balance equation for the particulate matter within the filter can be written as

$$
u \frac{d C}{d z}=-J_{p} \vartheta
$$

Here, $\vartheta$ represents the specific surface area of the filter $\left(\mathrm{m}^{2} / \mathrm{m}^{3}\right)$ and $u$ is the airstream velocity through the filter. Substituting Eq. (A1) into (A2) and integrating with respect to $C$, the following equation is obtained:

$$
C_{L}=C_{0} \exp \left(-\frac{\vartheta \Theta}{u} z\right)
$$

with

$$
\Lambda=\frac{\vartheta \Theta}{u}
$$

where $\Lambda$ is the called the filter coefficient (Bejan et al., 2004)

\section{APPENDIX B}

During the early moments of the deposition process, particles deposit directly onto the filter collectors (e.g., beds, fibers), but some time after, particles start depositing directly onto the particles previously deposited (Tanthapanichakoon et al., 2003). 
The temporal variation of particle deposition onto the filter collectors (early moments of deposition process) and onto previously deposited particles (later stage of filtration process) can be obtained based on Eqs. (13) and (14) with the particle concentration in the airstream within the filter given by Eq. (24). Therefore,

$$
\begin{aligned}
& \Phi_{c}^{*}=\frac{\alpha I_{p c} C_{0}^{*}}{k_{e}-\frac{k_{e} b_{1}}{b_{2}} \exp \left[\left(b_{1}-b_{2}\right) L^{*}\right]} \\
& \times\left[\frac{1}{b_{1}}\left[\exp \left(b_{1} L^{*}\right)-1\right]\right. \\
& \left.-\frac{b_{1}}{b_{2}^{2}}\left[\exp \left(b_{2} L^{*}\right)-1\right] \exp \left[\left(b_{1}-b_{2}\right) L^{*}\right]\right] \\
& \times\left[1-\exp \left(-\frac{k_{e}}{\alpha I_{p c}} \frac{\operatorname{Sh}_{c} \vartheta^{*}}{l_{c}^{*}} t^{*}\right)\right]
\end{aligned}
$$

$$
\begin{aligned}
& \Phi_{p}^{*}=\left[\frac{1}{b_{1}}\left[\exp \left(b_{1} L^{*}\right)-1\right]\right. \\
& \left.-\frac{b_{1}}{b_{2}^{2}}\left[\exp \left(b_{2} L^{*}\right)-1\right] \exp \left[\left(b_{1}-b_{2}\right) L^{*}\right]\right] \\
& \times \frac{C_{0}^{*}}{1-\frac{b_{1}}{b_{2}} \exp \left[\left(b_{1}-b_{2}\right) L^{*}\right]} \frac{\operatorname{Sh}_{p} \vartheta^{*}}{l_{c}^{*}} t^{*}
\end{aligned}
$$

with

$$
\mathrm{Sh}_{c}=\frac{l_{c} \Theta_{c}}{D} ; \quad S h_{p}=\frac{l_{c} \Theta_{p}}{D}
$$

The particulate matter transfer coefficients $\Theta_{c}$ and $\Theta_{p}$ can be obtained from the fitting of curves resulting from Eqs. (B1) and (B2) with corresponding experimental data of filters loaded with submicrometer particles. 